\title{
Modelling and Simulation of PQ Disturbance Based on Matlab
}

\author{
Wu Zhu, Wei-Ya Ma*, Yuan Gui, Hua-Fu Zhang \\ Shanghai University of Electric Power, 2103 pingliang road, Shanghai 200090, China
}

\begin{abstract}
In recent years, PQ (power quality) problem makes quality of power supply drop, which even triggers power accident, so PQ has attracted widespread attention. To improve and increase PQ, it needs to analyze and research on PQ disturbance systematically. The paper establishes models of short-term voltage change and harmonic disturbance with the method of setting fault and changing load based on simulation platform of Matlab. The simulation of the model gets wavelet of short-term voltage change and harmonic disturbance, the waveform is analyzed through Flourier transform. The simulation results and the theoretical analysis show that the model in this paper could simulate the voltage change and harmonic disturbance well, which can provide data and basis for detection and identification of $\mathrm{PQ}$ and further control measures.
\end{abstract}

Keywords: Power quality, modelling, simulation, Matlab

\section{Introduction}

Electric power is the main power used in modern industry, agriculture and daily life. With the development of industry, the scale of power system is expending, power quality disturbance have came with the wide use of nonlinear load. Power quality disturbance makes quality of power supply decline, which even causes power accident.

PQ (power quality) has aroused wide concern in recent years. PQ has great influence on safe and economic run of grid, reduce of energy consumption, guarantee of normal working of industry and scientific experiments. It's necessary to detect PQ disturbance to increase PQ, while the research on analysis and detection of the disturbance need wave form and data of the disturbance. Modelling and simulation of the PQ disturbance can get its wave form and data, which can be supplied for the research of PQ detect and analyze. At the same time, the waveform can help to understand PQ intuitively, so the modelling and simulation have high theoretical value, it is necessary to model the PQ disturbance.

Literature [1] modelled power system shorting fault by Matlab. It analyzes voltage and current of power system after fault, but this refers temporal voltage drop only, other PQ disturbance is not included, the model can not be used widely. Literature [2] modelled power system by Pscad/Emtdc to simulate various kinds of PQ disturbance, however it needs to lead voltage wave form to Matlab for the following analysis and detection. It needs to consider the interface transformation between Matlab and Pscad, which is fussy.

Simulink is a visual simulation tool of Matlab, it can be used in the simulation of power system and power electronics device. This paper builds PQ disturbance model based on Simulink of Matlab, it gets disturbance waveform of voltage change and harmonic disturbance through simulation. The waveforms are analyzed in the paper also.

\footnotetext{
* Manuscript received May 26, 2012; revised July 9, 2012.

Corresponding author. Tel.: +86-13482663718; E-mail address: maweiya8707@163.com.
} 


\section{Transient PQ Disturbance Modeling and Simulation}

The PQ disturbance can be divided into transient and steady disturbance. Transient PQ problems include voltage change and various transient phenomenons. Voltage change is phenomenon that Root Mean Square (RMS) of node voltage changes in a short time. Because of system failure or large load transformation, the voltage changes with time, including voltage sag, voltage swell and voltage interruption. Transient phenomenon is normally mean transient overvoltage, which can be divided into transient pulse and transient oscillation [3]. Voltage change is the most serious transient PQ problem affecting many electronic equipment's normal run [4], so this paper considers voltage change mainly

\subsection{Design of voltage change model and parameter setting}

Short fault often occurs in power system. It contains three phase shorting, two phase shorting, two phase earthing and single phase earthing. Most fault of transmission and distribution system are single phase earthing fault, which is the main reason of voltage sag [5]. Single phase earthing fault may make the power system voltage fluctuation happen, causing voltage sag, interruption, swell and current swell. The schematic diagram of phase A earthing in power system is as following:

When the line occurs phase A earthing, the fault line voltage to earth are [6]:

$$
\begin{aligned}
& U_{A-D}=0 \\
& U_{B-D}=U_{B}-U_{A}=\sqrt{3} U_{A} e^{-j 150^{\circ}} \\
& U_{C-D}=U_{C}-U_{A}=\sqrt{3} U_{A} e^{j 150^{\circ}}
\end{aligned}
$$

Among them, the $U_{A}, U_{B}, U_{C}$ are the voltage of phase A, B and C receptively. When phase A happens single phase earthing fault, the voltage to earth of phase $\mathrm{A}$ is equal to 0 , and the voltage of phase $\mathrm{B}, \mathrm{C}$ of the fault spot raise up to $3^{1 / 2}$ of the original.

Build the voltage disturbance model in Matlab on the basis of Fig. 1. Use the three-phase voltage source as power supply, separate parameter line as transmission line, and series RLC branch as load. The fault breaker supplies fault for the system.

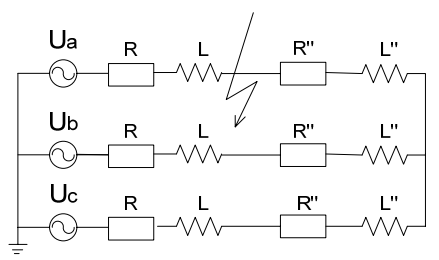

Fig. 1. Single phase earthing fault schematic diagram

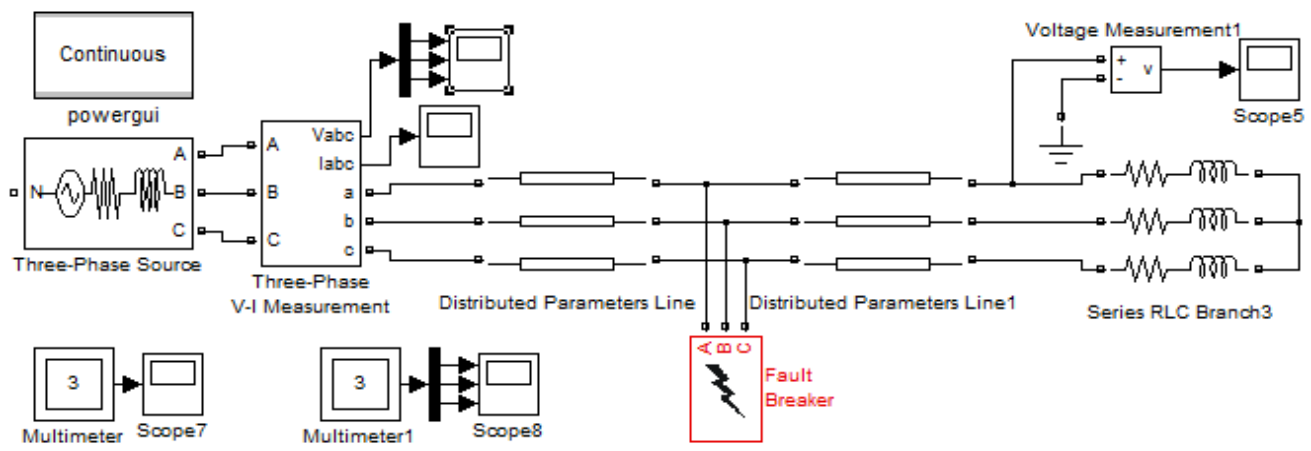

Fig. 2. Single phase earthing fault model

Choose the simulation time as $0.35 \mathrm{~s}$ and set a single-phase earthing fault between $0.11 \mathrm{~s}-0.15 \mathrm{~s}$. Parameters of the module in the model shown in Fig. 2 are set as following: Power module: set the voltage source as neutral grounding, the phase voltage of the three-phase voltage source as $380 \mathrm{~V}$ and power grid frequency as $50 \mathrm{~Hz}$; Transmission lines module: adopt the separate parameter line which is 40 
km long; Failure module: three-phase fault breaker is located between line 1 and line 2, it's set as phase A single earthing fault. The fault takes place in $0.11 \mathrm{~s}$ and it's cut off in $0.15 \mathrm{~s}$.Fault point resistance and fault point grounding resistance are selected for $0.001 \Omega$; Load module: select RL load, $R=10 \Omega, L=$ $0.005 \mathrm{H}$; Measurement modules: three-phase $V-I$ measurements are put in the circuit to measure voltage and current wavelet of the power side and the load side, the multimeter is put in the fault point to measure the voltage and current of it.

\subsection{Simulation result}

Select ode23tb as simulation algorithm and $0.35 \mathrm{~s}$ as simulation time in configuration parameter choice box. Then activate the simulation button, the voltages of phase A, B, C are shown in Fig. 3 .

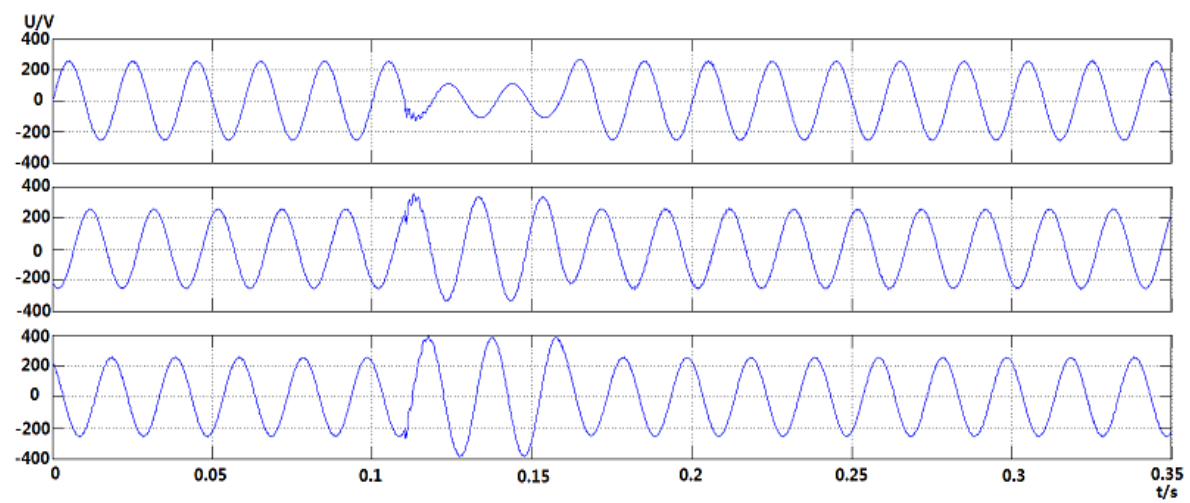

Fig. 3. Voltage of power side in single phase earthing fault

In Fig. 3, phase A voltage beside power drops with shake when single phase earthing happens in $0.11 \mathrm{~s}$, voltage of phase $\mathrm{B}$ and $\mathrm{C}$ rise. When fault generator disconnects in $0.15 \mathrm{~s}$, short-circuit fault is cut off. Because of the existence of dc (direct current) component in shorting current, the voltage of phase A is a little larger than normal voltage at this time. With the decline of dc component, voltage of phase A recovers to normal. When the fault is cut off, voltages of phase $\mathrm{B}, \mathrm{C}$ drop to normal.

Using a multimeter to detect current waveform of phase A, B and C showing in Fig. 4 (the colours of phase $\mathrm{A}$ is blue and $\mathrm{B}, \mathrm{C}$ are red). In Fig. 4, current of phase A, B, C are 0 before the single phase shorting fault. Current of phase $\mathrm{A}$ is no longer for 0 when phase A shorting happens, the current of phase A shakes greatly with the law of sine wave while current of phase B, C remain for 0 . Current of phase A drops to 0 quickly when the fault is cut off.

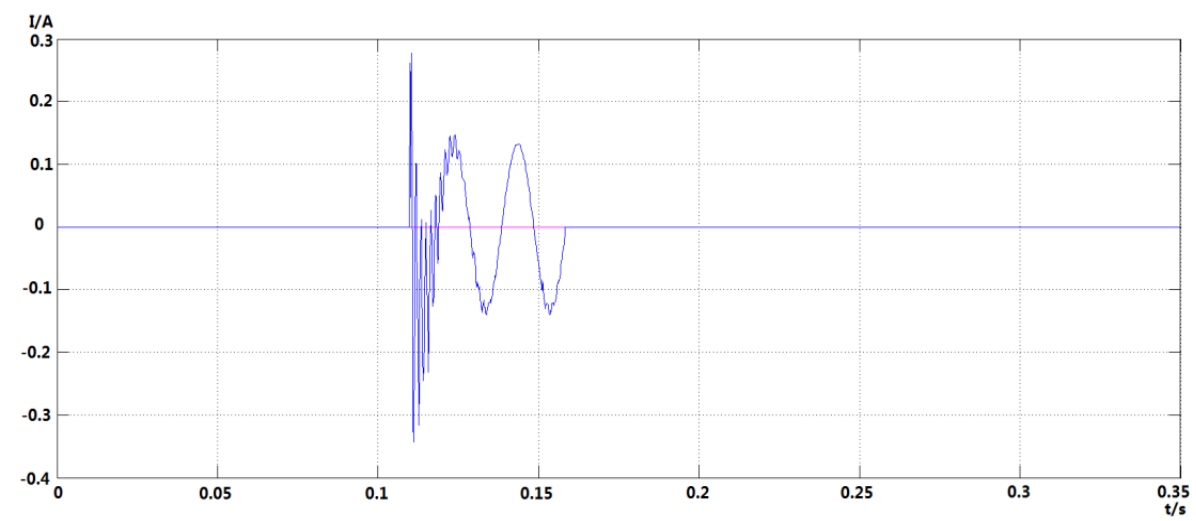

Fig. 4. Current waveform of fault point

Similarly, the voltage of phase A, B, C of fault point can be detected by a multimeter, the voltage is showing in Fig. 5:

As the figure shows, the voltage of phase $\mathrm{A}$ is interrupted when the three fault generator is closed in $0.11 \mathrm{~s}$. The voltage of phase $\mathrm{A}$ is 0 , while voltage of phase $\mathrm{B}$ and $\mathrm{C}$ rise. The voltage to earth of phase $\mathrm{B}$, $\mathrm{C}$ before fault is $160 \mathrm{~V}$, while the voltage rise to $270 \mathrm{~V}$ after fault, $270=160 \times 3^{1 / 2}$, which are fit to the 
formula (1), (2), (3). When the three phase fault generator is cut off in $0.15 \mathrm{~s}$, the voltages of phase A, B, $\mathrm{C}$ return to normal.

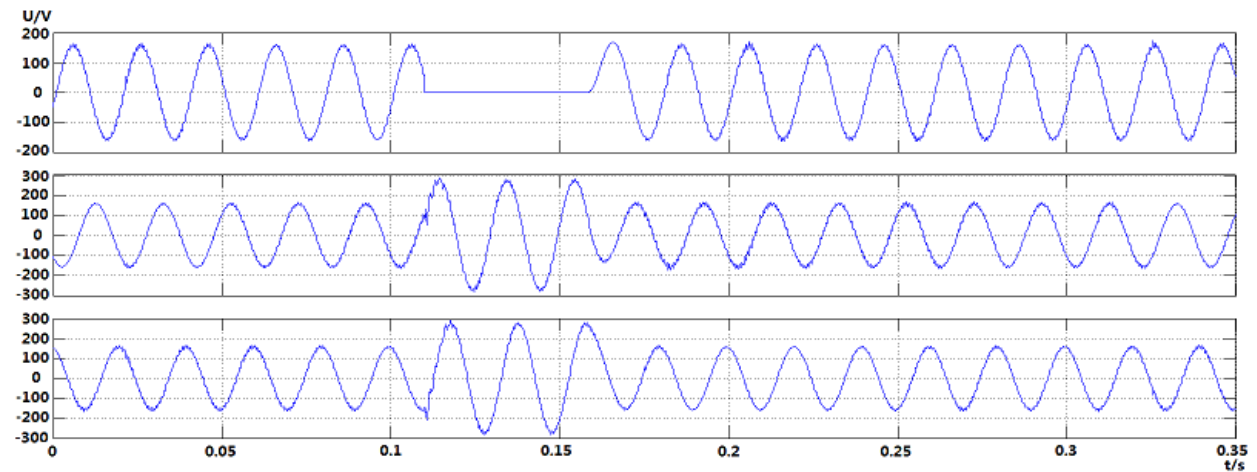

Fig. 5. The voltage waveform of fault point

\subsection{The factors influenced voltage change}

The difference in the distance from fault to load and the type of shorting fault will lead into the different extent of voltage changing.

The fault location will influence the extent of voltage change. The fault of model in Fig. 2 is set between $40 \mathrm{~m}$ and $40 \mathrm{~m}$, while move fault to the position between $20 \mathrm{~m}$ and $60 \mathrm{~m}$, that means setting the discrete parameter line 1 as $20 \mathrm{~m}$ long and discrete parameter line 2 as $60 \mathrm{~m}$ long. Through re-simulation can get phase A, B, C voltage beside power respectively after the fault happening. The rise and drop scale of voltage are changed as Fig. 6.

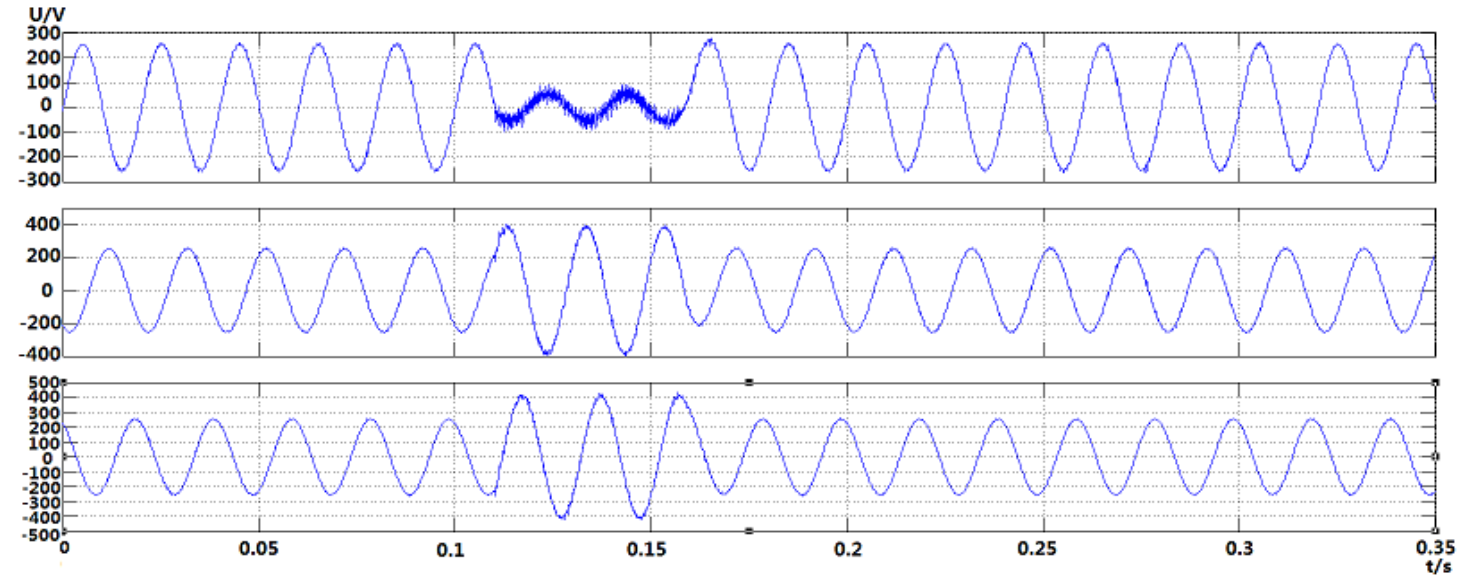

Fig. 6 . The voltage waveform beside power after the fault

In Fig. 3, single phase earthing leads voltage of A into dropping, the maximum voltage falls from 250 to 120 , the drop scale is 0.48 ; The fault leads voltage of phase $\mathrm{B}, \mathrm{C}$ into rising, the maximum voltage rises up from 250 to 340 , the rise scale is 1.36 . Fig. 6 shows the voltage of the power side after changing the fault position, voltage of phase A drops, the maximum voltage falls from 250 to 100 , the drop scale is 0.4 ; voltage of phase $\mathrm{B}, \mathrm{C}$ rise, the maximum voltage rise up from 250 to 400 , the rise scale is 1.6. Comparing with the Fig. 3, it's found that the shorter the distance between the load and fault point is, the more the voltage drops and the less the voltage rises.

The difference in type of fault will also result in different extent of voltage change. Set the three phase fault generator in model 3 as three phase shorting. The voltage of power side will be obtained through simulation, the voltage is shown in Fig. 7.

The voltage of phase A,B,C all happen sag, the maximum voltage falls from 250 to 210 , the drop scale is 0.807 . Comparing with the Fig. 3, it's found that the scale of voltage drop in three phase shorting is smaller than scale of voltage drop in single phase earthing. 


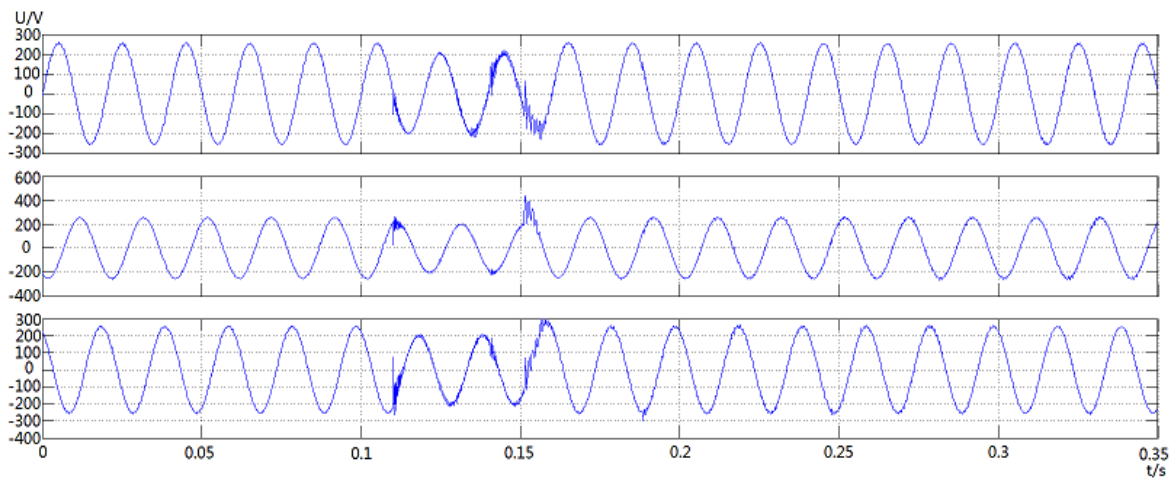

Fig. 7. Voltage waveform beside power in three phase shorting

The analysis above showing that different fault type and the fault location parameters will cause different voltage disturbance to power system. Three phase shorting and single phase earthing cause different scale of voltage drop and rise. The scale of voltage drop and rise caused by two phase shorting are also different form them, while this situation is not analyzed in this paper. Even in the same type of shorting fault, the different fault position will cause different sag and swell of voltage.

A short time voltage change is the most common PQ distribution in power system, the simulation result of model in Fig. 2 is consistent with the theory, it can simulate the voltage change well. This model can help to set up intuitive understanding for all sorts of voltage change and provide the basis for the identification and further control measures of the voltage change disturbance.

\section{Transient PQ Disturbance Modeling and Simulation}

\subsection{Design of voltage change model and parameter setting}

Steady PQ disturbance mainly includes three phase voltage unbalance, voltage fluctuation and flicker, voltage offset, frequency offset, waveform in the characteristic of distortion, such as harmonics, interharmonic and notch, among these disturbances harmonic wave does the greatest harm to power system especially.

With the wide application of various power electronic devices, they bring harmonic and interharmonic pollution into power system. Harmonic and inter-harmonic pollution do harm to the safety, stability and economic run of power system [7]. Building harmonic simulation model can analyze the harmonic pollution brought by power electronic devices in power system. The analysis has important theoretical and practical significance, therefore this paper will model and simulate harmonic disturbance mainly.

Taking three-phase bridge controllable rectifier circuit as an example, this paper analyzes the influence of power electronic equipment to the grid harmonics. Its principle diagram is shown as shown in Fig. 8, which chooses diode rectifier bridge as rectifier bridge.

Decompose the current of three-phase diode bridge rectifier with resistance load into Flourier series, taking phase A for example: make the middle point of negative half wave and positive half wave as time horizon:

$$
i_{a}=\sqrt{2} I_{1} \sin w t+\sum_{\substack{n=6 k \pm 1 \\ k=1,2,3, \ldots}}(-1)^{k} \sqrt{2} I_{n} \sin n w t
$$

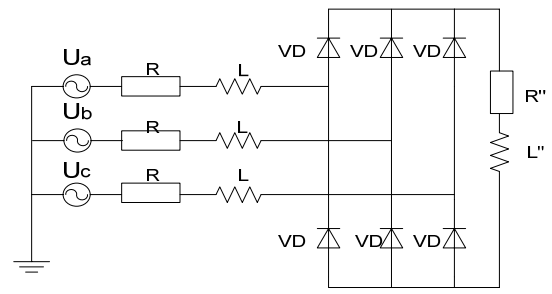

Fig. 8. Three-phase bridge controllable rectifier circuit schematic diagram 
Among them, the $I_{1}$ is RMS of base frequency current, $I_{n}$ is the RMS of the Nth times harmonic current. From the formula (4) can get base frequency current and the effective value of every harmonic are:

$$
i_{1}=\frac{\sqrt{6}}{\pi} I_{d}, \quad i_{n}=\frac{\sqrt{6}}{n \pi} \mathrm{I}_{d}, n=6 k \pm 1, k=1,2,3 \ldots
$$

$I_{d}$ is average value of output current. Visibly, the current contains only $6 \mathrm{k} \pm 1 \mathrm{st}$ ( $\mathrm{k}$ is a positive integer) times harmonic.

This paper aims to build a model applicable to analyze various disturbances. In the basis of the model of transient disturbance, taking off fault generator, changing the load to three-phase diode bridge rectifier according to Fig. 8, the harmonic disturbance model will be showed in Fig. 9. Three-phase diode bridge rectifier is a harmonic source.

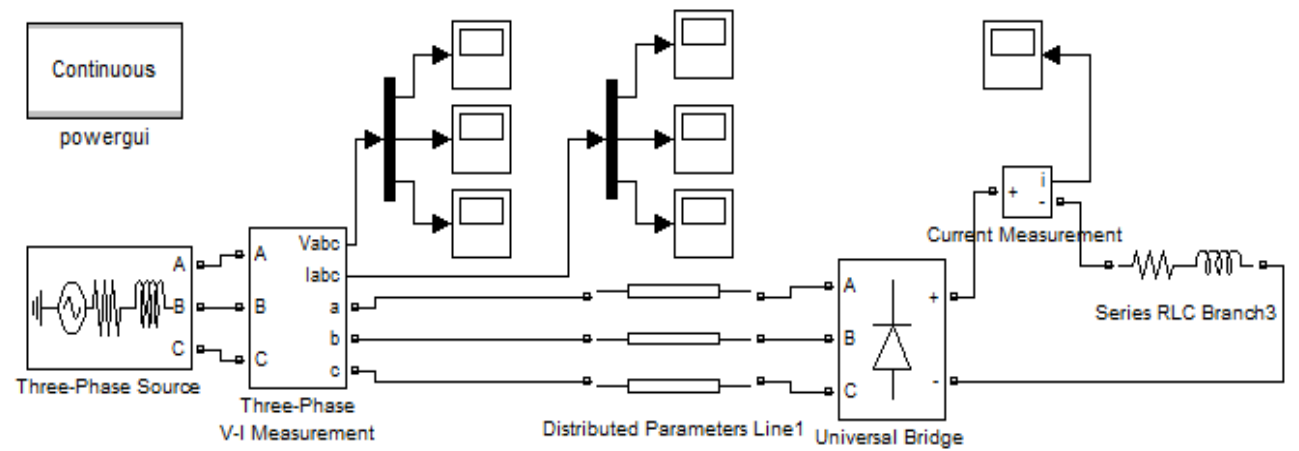

Fig. 9. Harmonic disturbance model

\subsection{Simulation result}

Similarly, select ode $23 \mathrm{tb}$ as simulation algorithm in configuration parameter and $0.35 \mathrm{~s}$ as simulation time. Then activate the simulation button, the voltage of phase $\mathrm{A}$ of power side and its Flourier analysis are shown in Fig. 10.

The basis frequency component of phase A voltage is 195.8, THD (total harmonic distortion) is $23.68 \%$, harmonic numbers are 5, 7, 11, 13 etc, that is $6 \mathrm{k} \pm 1$ st. It can be seen from the graph that harmonic RMS is inversely proportional to the number of the harmonic.

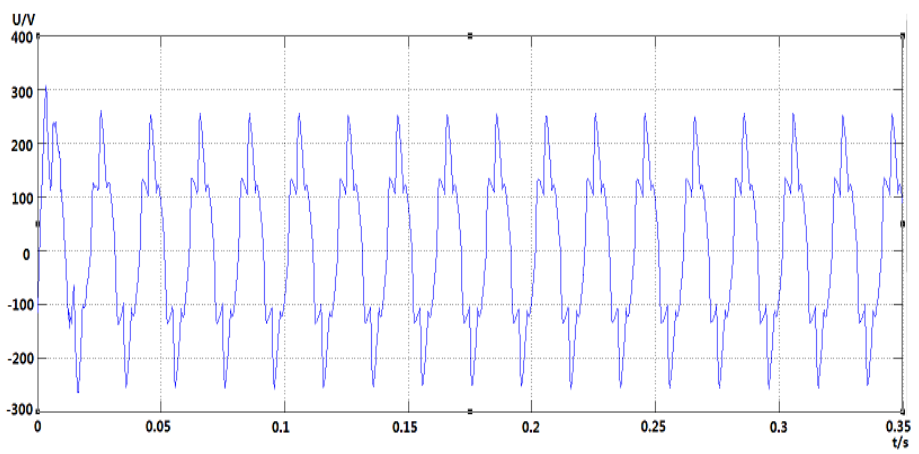

(a)

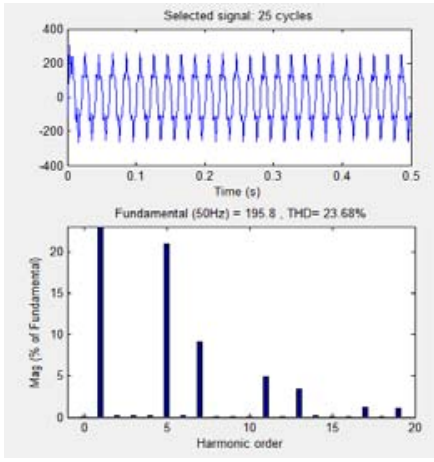

(b)

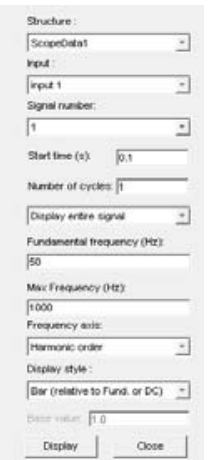

(b) Flourier analysis of the voltage

Fig. 10. (a) The voltage of phase A of power side

Current waveform of the power supply side is shown in Fig. 11 (a), its Fourier analysis is shown in Fig. 11 (b).

The basis frequency component of phase A current is 27.33 , THD (total harmonic distortion) is $6.00 \%$, harmonic numbers are $5,7,11,13 \mathrm{etc}$, that is $6 \mathrm{k} \pm 1$ st. It can be seen from the graph that harmonic RMS is inversely proportional to the number of the harmonic. 
From the Flourier analysis of voltage and current waveform of power side, it's found that rectifier equipments bring harmonic into power system, which makes voltage and current waveform occur distortion. The voltage and current beside the power side contains only $6 \mathrm{k} \pm 1 \mathrm{st}$ ( $\mathrm{k}$ is a positive integer) times harmonic, which is according with formula (5). It's demonstrated that this model can simulate harmonic disturbance well.

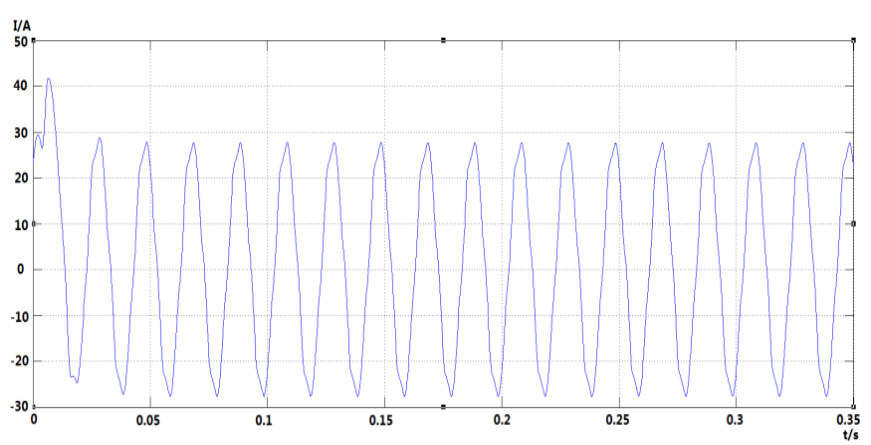

(a)

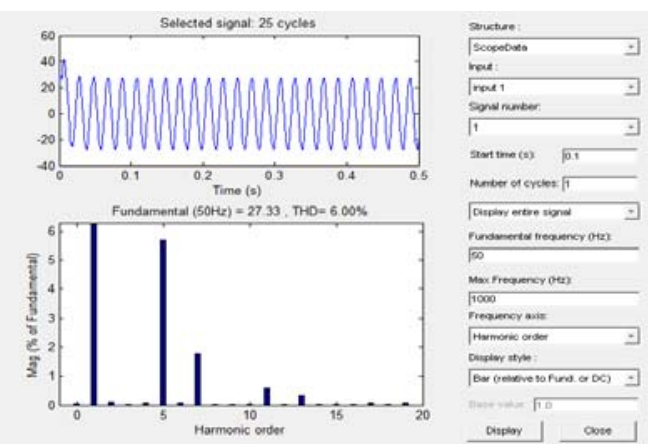

(b)

Fig. 11. (a) The current of phase A of power side (b) Flourier analysis of the current

\section{Conclusions}

The model built based on Matlab simulation platform in this paper is applicable to analyze voltage change and harmonic disturbance. The model is simple, efficient and highly applicable. The wavelet of voltage sag, voltage swell, and voltage interruption are getting from the simulation. The paper also analyzes the voltage and current contained harmonic through Flourier transformation. The simulation results and the theoretical analysis show that the model in this paper can simulate the voltage change and harmonic disturbance well, which can provide data and basis for detection and identification of PQ and further control measures.

\section{Acknowledgment}

This paper was supported by Shanghai Technology Innovation Project (10110502200), innovation Program of Shanghai Municipal Education Commission (11ZZ173).

\section{References}

[1] Yuan B, Chen J, Zhang W. Power transmission lines shorting analysis based on Matlab. Science Technology and Engineering, 2008; 8(15):4319-4320.

[2] He C. Powe quality disturbance classification based on the wavelet transform and the artificial neural network. Master thesis, Hunan University. Hunan, China; 2009.

[3] $\mathrm{Hu}$ M, Chen HG. Power quality and its analysis methods summarize. Power grid technology, 2000; 24(2):36-38.

[4] Shi J. Research on location of transient power quality disturbance. Master thesis, Hunan University. Hunan, China; 2008.

[5] Analysis and Control of Power Quality. Beijing: China Electric Power Press; 2010.

[6] Zhou YS, Peng Z, Wei XY, Kuang JH. Matlab simulation on single-phase-to-earth fault of small current grounding power system. Proceedings of the CSU-EPSA, 2009; 21(4):50-52.

[7] Pak LF, Dinavahi V, Chang G, Steurer M, Ribeiro PF. Real-time digital time-varying harmonic modeling and simulation techniques - IEEE task force on harmonics modeling and simulation. IEEE Transactions on Power Delivery, 2007; 22(2):1218-1227. 